\title{
Dynamic Analysis of Nonlinear Impulsive Neutral Nonautonomous Differential Equations with Delays
}

\author{
Jinxian Li \\ School of Mathematical Science, Shanxi University, Taiyuan 030006, China \\ Correspondence should be addressed to Jinxian Li; lijinxian@sxu.edu.cn
}

Received 6 January 2014; Accepted 26 February 2014; Published 1 April 2014

Academic Editor: Weiming Wang

Copyright (C) 2014 Jinxian Li. This is an open access article distributed under the Creative Commons Attribution License, which permits unrestricted use, distribution, and reproduction in any medium, provided the original work is properly cited.

A class of neural networks described by nonlinear impulsive neutral nonautonomous differential equations with delays is considered. By means of Lyapunov functionals and differential inequality technique, criteria on global exponential stability of this model are derived. Many adjustable parameters are introduced in criteria to provide flexibility for the design and analysis of the system. The results of this paper are new and they supplement previously known results. An example is given to illustrate the results.

\section{Introduction}

Many evolution processes in nature exhibit abrupt changes of states at certain moments. That was the reason for the development of the theory of impulsive differential equations and impulsive delay differential equations; see the monographs [1, 2]. But the theory of impulsive neutral differential equations is not well developed due to some theoretical and technical difficulties. For impulsive neutral differential equations, some existence results and oscillation criteria are obtained in [3-5] and some stability conditions are derived in [6]; for neural networks described by impulsive neutral differential equations with delays, the exponential stability results are obtained in [7-11], but their work focuses on the autonomous system. So in this paper, the exponential stability for neural networks described by nonlinear impulsive neutral nonautonomous differential equations with delays is considered.

The purpose of this paper is to study the stability of the following impulsive neural networks with variable coefficients and several time-varying delays:

$$
\begin{aligned}
\dot{x}_{i}(t)= & -b_{i}(t) x_{i}(t)+\sum_{j=1}^{n} a_{i j}(t) f_{i j}\left(x_{j}(t)\right) \\
& +\sum_{j=1}^{n} c_{i j}(t) g_{i j}\left(x_{j}\left(t-\tau_{i j}(t)\right)\right)
\end{aligned}
$$

$$
\begin{gathered}
+\sum_{j=1}^{n} d_{i j}(t) h_{i j}\left(x_{j}^{\prime}\left(t-\widehat{\tau}_{i j}(t)\right)\right) \\
+k_{i}(t), \quad \text { a.e. } t>0, t \neq t_{k}, \\
x_{i}\left(t^{+}\right)=I_{i k}\left(x_{i}(t)\right)+J_{i k}\left(x_{i}\left(t-\varsigma_{i}(t)\right)\right)+K_{i k}(t), \\
\quad t=t_{k}, \quad i=1,2, \ldots, n ; \quad k=1,2, \ldots,
\end{gathered}
$$

where $n$ corresponds to the number of units in a neural network; for $i, j=1,2, \ldots, n, x_{i}(t)$ denotes the potential of cell $i$ at time $t ; 0 \leq \tau_{i j}(t), \widehat{\tau}_{i j}(t), c_{i}(t) \leq \tau$ correspond to the transmission delays. (1a) (called continuous part) describes the continuous evolution processes of the neural networks. For $i, j=1,2, \ldots, n, a_{i j}(t), c_{i j}(t)$, and $d_{i j}(t)$ denote the strengths of connectivity between cells $i$ and $j$ at time $t$, respectively; $f_{i j}, g_{i j}, h_{i j}$ show how the $i$ th neuron reacts to the input; $k_{i}(t)$ is the external bias on the $i$ th at time $t$. (1b) (called discrete part) describes that the evolution processes experience abrupt change of states at the moments of $t_{k}$ (called impulsive moments); for $i=1,2, \ldots, n, k=1,2, \ldots$, the fixed moment $t_{k}$ satisfies $t_{1}<t_{2}<\cdots<t_{k}<\cdots$, and $\lim _{k \rightarrow \infty} t_{k}=\infty$; $I_{i k}$ represents impulsive perturbations of $i$ th unit at time $t_{k} ; J_{i k}$ represents impulsive perturbations of $i$ th unit at time $t_{k}$, which is caused by the transmission delays; $K_{i k}\left(t_{k}\right)$ represents the external impulsive input at time $t_{k}$. 
The theory on linear matrix inequality (LMI) or $M$ Matrix provides effective methods for the analysis of exponential stability of autonomous neural networks. See $[7,9,10]$ and the reference therein. But for nonautonomous neural networks, it is invalid. Differential inequalities are important tools for investigating the stability of impulsive differential equations. See $[7,8,12,13]$ and the reference therein. The method in this paper is partially motivated by the work in [7].

In this paper, we will investigate the global exponential stability of the nonautonomous neural networks and focus on the effect of impulse on the dynamic behavior of (1a) and (1b). The results do not require the boundedness of $\left\{t_{k}-t_{k-1}\right\}$ and the differentiability of $\tau_{i j}$. So they are new and complement previously known results.

For a continuous function $a(t)$, we denote

$$
\begin{array}{cc}
a^{+}(t)=\max \{0, a(t)\}, & a^{-}(t)=\min \{0, a(t)\}, \\
a\left(t^{+}\right)=\lim _{s \rightarrow t^{+}} a(s), & a\left(t^{-}\right)=\lim _{s \rightarrow t^{-}} a(s) .
\end{array}
$$

Define

$$
\begin{gathered}
R^{+}=[0, \infty), \quad N=\{1,2, \ldots, n\}, \quad N^{*}=\{1,2, \ldots\}, \\
C(\Omega, R)=\{\psi: \Omega \longrightarrow R \mid \psi \text { is continous, } \Omega \subset R\}, \\
C B(\Omega, R)=\{\psi \in C(\Omega, R) \mid \psi \text { is bounded }\}, \\
P C([-\tau, 0], R) \\
=\{\psi:[-\tau, 0] \longrightarrow R \\
\mid \psi\left(t^{-}\right)=\psi(t), \text { for } t \in[-\tau, 0], \psi\left(t^{+}\right) \\
\text {exists on } R \text { and } \psi\left(t^{+}\right)=\psi(t) \\
\text { for all but at most a finite } \\
\text { number of points on }[-\tau, 0] .\}, \\
P C^{1}([-\tau, 0], R) \\
=\{\psi \in P C([-\tau, 0], R) \\
\mid \psi^{\prime}\left(t^{+}\right) \text {and } \psi^{\prime}\left(t^{-}\right) \text {exist, } \psi^{\prime}(t)=\psi^{\prime}\left(t^{-}\right) \\
\text {for } t \in[-\tau, 0] \text { and } \psi^{\prime}\left(t^{+}\right)=\psi^{\prime}(t) \\
\text { for all but at most a finite } \\
\text { number of points on }[-\tau, 0] .\}, \\
P C\left([-\tau, 0], R^{n}\right) \\
=\left\{\widehat{\psi}=\left(\psi_{1}, \psi_{2}, \ldots, \psi_{n}\right)^{T}\right.
\end{gathered}
$$

$$
\begin{aligned}
& \left.\mid \psi_{i} \in P C([-\tau, 0], R), i \in N .\right\}, \\
& P C^{1}\left([-\tau, 0], R^{n}\right) \\
& =\left\{\widehat{\psi}=\left(\psi_{1}, \psi_{2}, \ldots, \psi_{n}\right)^{T}\right. \\
& \left.\quad \mid \psi_{i} \in P C^{1}([-\tau, 0], R), i \in N .\right\} .
\end{aligned}
$$

For any $\phi \in P C([-\tau, 0], R), \widehat{\phi} \in P C^{1}([-\tau, 0], R), \psi=\left(\psi_{1}\right.$, $\left.\psi_{2}, \ldots, \psi_{n}\right)^{T} \in P C\left([-\tau, 0], R^{n}\right)$, and $\widehat{\psi}=\left(\widehat{\psi}_{1}, \widehat{\psi}_{2}, \ldots, \widehat{\psi}_{n}\right)^{T} \epsilon$ $P C^{1}\left([-\tau, 0], R^{n}\right)$, define $\|\cdot\|_{\tau},\|\cdot\|_{1 \tau},\|\cdot\|_{\tau}^{n}$, and $\|\cdot\|_{1 \tau}^{n}$ as

$$
\begin{gathered}
\|\phi\|_{\tau}=\sup _{-\tau \leq s \leq 0}|\phi(s)|, \quad\|\hat{\phi}\|_{1 \tau}=\max \left\{\|\hat{\phi}\|_{\tau^{\prime}}\left\|\hat{\phi}^{\prime}\right\|_{\tau}\right\}, \\
\|\psi\|_{\tau}^{n}=\max _{1 \leq i \leq n}\left\|\psi_{i}\right\|_{\tau^{\prime}}, \quad\|\widehat{\psi}\|_{1 \tau}^{n}=\max _{1 \leq i \leq n}\left\|\widehat{\psi}_{i}\right\|_{1 \tau},
\end{gathered}
$$

respectively.

For convenience, the following conditions are listed.

$\left(\mathrm{H}_{1}\right)$ For $i, j \in N, b_{i} \in C\left(R^{+}, R^{+}\right), a_{i j} \in C\left(R^{+}, R\right)$, and $c_{i j}, d_{i j} \in C B\left(R^{+}, R\right), f_{i j}, g_{i j}, h_{i j} \in C(R, R)$.

$\left(\mathrm{H}_{2}\right)$ There are positive constants $F_{i j}, G_{i j}, H_{i j}, i, j \in N$, such that

$$
\begin{aligned}
& \left|f_{i j}(u)-f_{i j}(v)\right| \leq F_{i j}|u-v|, \\
& \left|g_{i j}(u)-g_{i j}(v)\right| \leq G_{i j}|u-v|, \\
& \left|h_{i j}(u)-h_{i j}(v)\right| \leq H_{i j}|u-v|,
\end{aligned}
$$

for all $u, v \in R$.

$\left(\mathrm{H}_{3}\right)$ There exist positive constants $I_{i k}^{*}$ and $J_{i k}^{*}, i \in N, k \in$ $N^{*}$, such that

$$
\begin{gathered}
\left|I_{i k}(u)-I_{i k}(v)\right| \leq I_{i k}^{*}|u-v|, \\
\left|J_{i k}(u)-J_{i k}(v)\right| \leq J_{i k}^{*}|u-v|, \\
\max _{i \in N, k \in N^{*}} I_{i k}^{*}+\max _{i \in N, k \in N^{*}} J_{i k}^{*}<1,
\end{gathered}
$$

for all $u, v \in R$.

$\left(\mathrm{H}_{4}\right)$ There exist positive constants $p_{i}, q_{i}, i \in N$ and $\sigma$ such that

$$
\begin{aligned}
& p_{i} b_{i}(t)-\sum_{j=1}^{n} p_{j} F_{i j} a_{i j}^{+}(t) \\
& -\sum_{j=1}^{n}\left(p_{j} G_{i j} c_{i j}^{+}(t)+q_{j} H_{i j} d_{i j}^{+}(t)\right) \geq \sigma>0, \\
& q_{i}-p_{i} b_{i}(t)-\sum_{j=1}^{n} p_{j} F_{i j} a_{i j}^{+}(t) \\
& -\sum_{j=1}^{n}\left(p_{j} G_{i j} j_{i j}^{+}(t)+q_{j} H_{i j} d_{i j}^{+}(t)\right) \geq \sigma>0,
\end{aligned}
$$

for $t \in[0, \infty), i \in N$. 
We assume that (1a) and (1b) are with the following initial conditions:

$$
x(s)=\phi(s), \quad s \in[-\tau, 0],
$$

where $\phi \in P C\left([-\tau, 0], R^{n}\right)$. According to [13], the initial value problems (1a), (1b), and (8) have the unique solution $x(t, \phi)$ under assumptions $\left(\mathrm{H}_{2}\right)$ and $\left(\mathrm{H}_{3}\right)$.

Definition 1. A function $x(t)=\left(x_{1}(t), x_{2}(t), \ldots, x_{n}(t)\right)^{T}$ is said to be a solution of (la) and (1b) on $[-\tau, \infty)$ if for $i \in N$,

(i) $x_{i}(t)$ is absolutely continuous on each interval $\left(0, t_{1}\right)$ and $\left(t_{k}, t_{k+1}\right), k \in N^{*}$;

(ii) for any $t_{k}, k \in N^{*}, x_{i}\left(t_{k}^{+}\right)$and $x_{i}\left(t_{k}^{-}\right)$exist and $x_{i}\left(t_{k}^{-}\right)=$ $x_{i}\left(t_{k}\right)$;

(iii) $x(t)$ satisfies (1a) for almost everywhere in $[0, \infty)$ and satisfies (1b) for every $t=t_{k}, k \in N^{*}$.

Obviously, a solution $X(t)=\left(x_{1}(t), x_{2}(t), \ldots, x_{n}(t)\right)^{T}$ of (1a) and (1b) is continuous at $t \neq t_{k}$ and discontinuous at $t=t_{k}$. Furthermore, $X^{\prime}(t)=\left(x_{1}^{\prime}(t), x_{2}^{\prime}(t), \ldots, x_{n}^{\prime}(t)\right)^{T}$ has discontinuities of the first kind at the fixed impulsive moments $t_{k}$ and some moments $\bar{t} \in\left(t_{k}, t_{k+1}\right), k \in N^{*}$. Denote $X^{\prime}\left(t_{k}\right)=X^{\prime}\left(t_{k}^{-}\right), X^{\prime}(\bar{t})=X^{\prime}(\bar{t})$.

Definition 2. Let $X(t)=\left(x_{1}(t), x_{2}(t), \ldots, x_{n}(t)\right)^{T}$ and $Y(t)=$ $\left(y_{1}(t), y_{2}(t), \ldots, y_{n}(t)\right)^{T}$ be two solutions of (1a), (1b), and (8) with $\phi=\varphi$ and $\phi=\psi$, respectively, where $\varphi$ and $\psi \epsilon$ $P C\left([-\tau, 0], R^{n}\right)$. If there exist $\alpha>0$ and $M>1$ such that

$$
\left|x_{i}(t)-y_{i}(t)\right| \leq M\|\varphi-\psi\|_{1 \tau}^{n} e^{-\alpha t}, \quad \forall t>0, i \in N
$$

then (1a) and (1b) are said to be globally exponentially stable.

\section{The Main Result}

To study the exponential stability of (1a) and (1b), we need the following lemma.

Lemma 3. Assume that $\left(H_{1}\right)$ and $\left(H_{4}\right)$ hold and there exist nonnegative vector functions $\left(V_{1}(t), V_{2}(t), \ldots, V_{n}(t)\right)^{T}$ and
$\left(W_{1}(t), W_{2}(t), \ldots, W_{n}(t)\right)^{T} \in P C\left([-\tau, 0], R^{n}\right)$, where $V_{i}(t)$ is continuous at $t \neq t_{k}\left(k \in N^{*}\right)$, such that

$$
\begin{aligned}
D^{-} V_{i}\left(t^{-}\right) \leq & -b_{i}(t) V_{i}\left(t^{-}\right)+\sum_{j=1}^{n} a_{i j}^{+}(t) F_{i j} V_{j}\left(t^{-}\right) \\
& +\sum_{j=1}^{n} c_{i j}^{+}(t) G_{i j}\left\|V_{j t^{-}}\right\|_{\tau}+\sum_{j=1}^{n} d_{i j}^{+}(t) H_{i j}\left\|W_{j t^{-}}\right\|_{\tau^{\prime}}
\end{aligned}
$$

$$
\begin{aligned}
W_{i}\left(t^{+}\right) \leq & b_{i}(t) V_{i}\left(t^{+}\right)+\sum_{j=1}^{n} a_{i j}^{+}(t) F_{i j} V_{j}\left(t^{+}\right) \\
& +\sum_{j=1}^{n} c_{i j}^{+}(t) G_{i j}\left\|V_{j t^{+}}\right\|_{\tau}+\sum_{j=1}^{n} d_{i j}^{+}(t) H_{i j}\left\|W_{j t^{+}}\right\|_{\tau},
\end{aligned}
$$

$$
V_{i}\left(t_{k}^{+}\right) \leq I_{i k}^{*} V_{i}\left(t_{k}\right)+J_{i k}^{*} V_{i}\left(t_{k}-\varsigma_{i}\left(t_{k}\right)\right),
$$

for $t>0, i \in N, k \in N^{*}$. Then for all $t \geq 0$ and $i \in N$, there exists a positive constant $L$ such that

$$
V_{i}(t) \leq L \sum_{l=1}^{n} \max \left\{\left\|V_{l 0}\right\|_{\tau},\left\|W_{l 0}\right\|_{\tau}\right\} e^{-\left(\lambda^{*}-\mu\right) t},
$$

where $\lambda^{*}$ and $\mu$ are defined, respectively, as

$$
\begin{aligned}
& \lambda^{*}=\min \left\{\lambda_{i}^{*}, \hat{\lambda}_{i}^{*} \mid i \in N\right\}, \\
& \lambda^{*}+\frac{1}{\tau} \ln \frac{\max _{i \in N, k \in N^{*}} J_{i k}^{*}}{1-\max _{i \in N, k \in N^{*}} I_{i k}^{*}} \leq \mu \leq \lambda^{*}, \\
& \lambda_{i}^{*}=\inf _{t \geq 0}\{\lambda(t)>0, \lambda(t) \\
& -\left[b_{i}(t)-\frac{1}{p_{i}} \sum_{j=1}^{n} p_{j} F_{i j} a_{i j}^{+}(t)\right] \\
& +\frac{1}{p_{i}} \sum_{j=1}^{n}\left(p_{j} G_{i j} c_{i j}^{+}(t)+q_{j} H_{i j} d_{i j}^{+}(t)\right) \\
& \left.\times e^{\lambda(t) \tau}=0\right\}>0, \\
& \widehat{\lambda}_{i}^{*}=\inf _{t \geq 0}\{\lambda(t)>0, \\
& -\left[1-\frac{p_{i}}{q_{i}} b_{i}(t)-\frac{1}{q_{i}} \sum_{j=1}^{n} p_{j} F_{i j} a_{i j}^{+}(t)\right] \\
& +\frac{1}{q_{i}} \sum_{j=1}^{n}\left(p_{j} G_{i j} c_{i j}^{+}(t)+q_{j} H_{i j} d_{i j}^{+}(t)\right) \\
& \left.\times e^{\lambda(t) \tau}=0\right\}>0 .
\end{aligned}
$$

Proof. By the similar analysis in [14, Lemma 4.1], we can deduce that $\lambda_{i}^{*}$ and $\hat{\lambda}_{i}^{*}$ exist uniquely and $\lambda_{i}^{*}>0, \hat{\lambda}_{i}^{*}>0$ under the assumption of $\left(\mathrm{H}_{1}\right)$ and $\left(\mathrm{H}_{4}\right)$. Consequently, $\lambda^{*}>$ 
0 . Choose a positive constant $\theta$ such that $\min \left\{p_{i}, q_{i} \mid i \epsilon\right.$ $N\} \theta>1$. Let

$$
\begin{gathered}
\Phi_{i}(t)=\max \left\{\frac{1}{p_{i}} V_{i}(t), \frac{1}{q_{i}} W_{i}(t)\right\}, \\
\Psi(t)=\theta \sum_{l=1}^{n} \max \left\{\left\|V_{l 0}\right\|_{\tau},\left\|W_{l 0}\right\|_{\tau}\right\} e^{-\left(\lambda^{*}-\mu\right) t},
\end{gathered}
$$

$i \in N$.

Then for all $t \in[-\tau, 0]$ and $\gamma>1$, we have

$$
\gamma \Psi(t)=\gamma \theta \sum_{l=1}^{n} \max \left\{\left\|V_{l 0}\right\|_{\tau},\left\|W_{l 0}\right\|_{\tau}\right\} e^{-\left(\lambda^{*}-\mu\right) t}>\Phi_{i}(t) .
$$

Then

$$
\Phi_{i}(t)<\gamma \Psi(t), \quad \forall t \in[0, \infty), i \in N .
$$

For the sake of contradiction, assume that there exist $i \in N$ and $\bar{t}>0$ such that

$$
\begin{array}{r}
\Phi_{i}\left(\bar{t}^{+}\right) \geq \gamma \Psi(\bar{t}), \quad \Phi_{j}(t)<\gamma \Psi(t), \\
\text { for } t \in[0, \bar{t}), \quad j \in N .
\end{array}
$$

From (17), we have

$$
\begin{aligned}
\left\|V_{j \bar{t}}\right\|_{\tau} & =p_{j} \sup _{-\tau \leq \theta \leq 0} \frac{1}{p_{j}} V_{j}(\bar{t}+\theta) \\
& \leq p_{j} \sup _{-\tau \leq \theta \leq 0} \gamma \Psi(\bar{t}+\theta) \leq \gamma p_{j} \Psi(\bar{t}-\tau) ;
\end{aligned}
$$

similarly,

$$
\left\|W_{j \bar{t}}\right\|_{\tau} \leq \gamma q_{j} \Psi(\bar{t}-\tau) .
$$

Then we have the following cases.

(I) $\left(1 / p_{i}\right) V_{i}\left(\bar{t}^{+}\right) \geq \gamma \Psi(\bar{t})$; then we have the following subcases.

(i) $\bar{t} \neq t_{k}, k \in N^{*}$. So $V_{i}(t)$ is continuous at $\bar{t}$. By (17), we have

$$
\frac{1}{p_{i}} V_{i}(\bar{t})=\gamma \Psi(\bar{t}), \quad \frac{1}{p_{i}} D^{-} V_{i}(\bar{t})>\gamma \Psi^{\prime}(\bar{t}) .
$$

From $\left(\mathrm{H}_{4}\right),(17)-(19)$, and the definition of $\lambda^{*}$, we have

$$
\begin{aligned}
& \frac{1}{p_{i}} D^{-} V_{i}(\bar{t})-\gamma \Psi^{\prime}(\bar{t}) \\
& \leq-\gamma b_{i}(\bar{t}) \Psi(\bar{t})+\sum_{j=1}^{n} \frac{p_{j}}{p_{i}} \gamma a_{i j}^{+}(\bar{t}) F_{i j} \Psi(\bar{t}) \\
& +\sum_{j=1}^{n} \gamma\left(\frac{p_{j}}{p_{i}} c_{i j}^{+}(\bar{t}) G_{i j}+\frac{q_{j}}{p_{i}} d_{i j}^{+}(\bar{t}) H_{i j}\right) \\
& \times \Psi(\bar{t}-\tau)+\gamma \lambda^{*} \Psi(\bar{t})<0,
\end{aligned}
$$

which is a contradiction with (20). (ii) There exists a $k_{0} \in N^{*}$ such that $\bar{t}=t_{k_{0}}$. By (17), we have

$$
\frac{1}{p_{i}} V_{i}(\bar{t}) \leq \gamma \Psi(\bar{t}) \leq \frac{1}{p_{i}} V_{i}\left(\bar{t}^{+}\right) .
$$

Noting $\left(1 / p_{i}\right) V_{i}\left(\bar{t}^{+}\right) \neq\left(1 / p_{i}\right) V_{i}\left(\bar{t}^{-}\right)$, we have $\left(1 / p_{i}\right) V_{i}\left(\bar{t}^{-}\right)<$ $\gamma \Psi(\bar{t})$ or $\gamma \Psi(\bar{t})<\left(1 / p_{i}\right) V_{i}\left(\bar{t}^{+}\right)$. Without loss of generality, we assume that $\gamma \Psi(\bar{t})<\left(1 / p_{i}\right) V_{i}\left(\bar{t}^{+}\right)$. From (10c) and (22), we get that

$$
\gamma \Psi(\bar{t})<\frac{1}{p_{i}} V_{i}\left(\bar{t}^{+}\right) \leq \gamma\left(I_{i k_{0}}^{*}+J_{i k_{0}}^{*} e^{\left(\lambda^{*}-\mu\right) \tau}\right) \Psi(\bar{t}) .
$$

Simplifying (23), we obtain $\mu<\lambda^{*}+(1 / \tau) \ln \left(J_{i k_{0}}^{*} /\left(1-I_{i k_{0}}^{*}\right)\right)$, which contradict (12).

If (I) does not hold, then

(II)

$$
\begin{gathered}
\frac{1}{q_{i}} W_{i}\left(\bar{t}^{+}\right) \geq \gamma \Psi(\bar{t}), \quad \frac{1}{q_{j}} W_{j}(t)<\gamma \Psi(t), \\
\frac{1}{p_{j}} V_{j}(t)<\gamma \Psi(t)
\end{gathered}
$$

for $t \in[0, \bar{t}), \quad j \in N$.

Then from (10b) and (17)-(19), we have

$$
\begin{aligned}
0 \leq & -W_{i}\left(\bar{t}^{+}\right)+b_{i}(\bar{t}) V_{i}\left(\bar{t}^{+}\right)+\sum_{j=1}^{n} a_{i j}^{+}(\bar{t}) F_{i j} V_{j}\left(\bar{t}^{+}\right) \\
+ & \sum_{j=1}^{n} c_{i j}^{+}(\bar{t}) G_{i j}\left\|V_{j t^{+}}\right\|_{\tau}+\sum_{j=1}^{n} d_{i j}^{+}(\bar{t}) H_{i j}\left\|W_{j \bar{t}^{+}}\right\|_{\tau^{\prime}} \\
\leq & \gamma \Psi(\bar{t})\left[-q_{i}+p_{i} b_{i}(\bar{t})+\sum_{j=1}^{n} p_{j} a_{i j}^{+}(\bar{t}) F_{i j}\right. \\
& \left.+\sum_{j=1}^{n}\left(p_{j} c_{i j}^{+}(\bar{t}) G_{i j}+q_{j} d_{i j}^{+}(\bar{t}) H_{i j}\right) e^{\lambda^{*} \tau}\right]<0,
\end{aligned}
$$

which is a contradiction.

From (I) and (II), (16) holds. Letting $\gamma \rightarrow 1^{+}$in (16), we have

$$
\Phi_{i}(t) \leq \Psi(t), \quad \forall t \in[0, \infty), i \in N .
$$

So $\left(1 / p_{i}\right) V_{i}(t) \leq \Psi(t)$ for all $t \in[0, \infty), i \in N$. Let $L=$ $\max _{i \in N}\left\{\theta p_{i}\right\}$; then for $t \geq 0$ and $i \in N$, we have

$$
V_{i}(t) \leq L \sum_{l=1}^{n} \max \left\{\left\|V_{l 0}\right\|_{\tau},\left\|W_{l 0}\right\|_{\tau}\right\} e^{-\left(\lambda^{*}-\mu\right) t} .
$$

The proof of Lemma 3 is complete.

Theorem 4. Assume that $\left(H_{1}\right)-\left(H_{4}\right)$ hold. Then systems (1a) and (1b) are globally exponentially stable. 
Proof. Let $X(t)=\left(x_{1}(t), x_{2}(t), \ldots, x_{n}(t)\right)^{T}$ and $Y(t)=$ $\left(y_{1}(t), y_{2}(t), \ldots, y_{n}(t)\right)^{T}$ be solutions of (1a), (1b), and (8) with $\phi=\varphi$ and $\phi=\psi$, respectively. Let

$$
\begin{array}{r}
V_{i}(t)=\left|x_{i}(t)-y_{i}(t)\right|, \quad W_{i}(t)=\left|x_{i}^{\prime}(t)-y_{i}^{\prime}(t)\right|, \\
t \in R^{+}, \quad i \in N .
\end{array}
$$

By (1a) and (1b), for $i \in N$, we have

$$
\begin{aligned}
D^{-} V_{i}\left(t^{-}\right) \leq & -b_{i}(t) V_{i}\left(t^{-}\right)+\sum_{j=1}^{n} a_{i j}^{+}(t) F_{i j} V_{j}\left(t^{-}\right) \\
& +\sum_{j=1}^{n} c_{i j}^{+}(t) G_{i j}\left\|V_{j t^{-}}\right\|_{\tau} \\
& +\sum_{j=1}^{n} d_{i j}^{+}(t) H_{i j}\left\|W_{j t^{-}}\right\|_{\tau^{\prime}} \quad t>0, \\
W_{i}\left(t^{+}\right) \leq & b_{i}(t) V_{i}\left(t^{+}\right) \\
& +\sum_{j=1}^{n} a_{i j}^{+}(t) F_{i j} V_{j}\left(t^{+}\right)+\sum_{j=1}^{n} c_{i j}^{+}(t) G_{i j}\left\|V_{j t^{+}}\right\|_{\tau} \\
& +\sum_{j=1}^{n} d_{i j}^{+}(t) H_{i j}\left\|W_{j t^{+}}\right\|_{\tau^{\prime}} t>0 .
\end{aligned}
$$

By (1b) and $\left(\mathrm{H}_{3}\right)$, we have

$$
V_{i}\left(t_{k}^{+}\right)=\left|x_{i}\left(t_{k}^{+}\right)-y_{i}\left(t_{k}^{+}\right)\right| \leq I_{i k}^{*} V_{i}\left(t_{k}\right)+J_{i k}^{*} V_{i}\left(t_{k}-\varsigma_{i}\left(t_{k}\right)\right) \text {. }
$$

By (29)-(31) and Lemma 3, there exists a positive constant $M$ such that

$$
\begin{aligned}
V_{i}(t) & \leq M \sum_{l=1}^{n} \max \left\{\left\|V_{l 0}\right\|_{\tau},\left\|W_{l 0}\right\|_{\tau}\right\} e^{-\left(\lambda^{*}-\mu\right) t} \\
& \leq M n\|\phi-\psi\|_{1 \tau}^{n} e^{-\left(\lambda^{*}-\mu\right) t}
\end{aligned}
$$

where $\lambda^{*}$ and $\mu$ are defined in (12).

Remark 5. For autonomous system, the exponential stability of the zero solution of (1a) with $x_{i}\left(t_{k}^{+}\right)=I_{i k}\left(x_{1}\left(t_{k}\right)\right.$, $\left.\ldots, x_{n}\left(t_{k}\right)\right), k \in N^{*}$, is considered in [7]. But the results require that $\left\{t_{k}-t_{k-1}\right\}$ is bounded.

When there is no impulse in systems (1a) and (1b), (1a) and (1b) reduce to the following model which has been studied in $[9,10]$ :

$$
\begin{aligned}
\dot{x}_{i}(t)= & -b_{i}(t) x_{\mathrm{i}}(t)+\sum_{j=1}^{n} a_{i j}(t) f_{i j}\left(x_{j}(t)\right) \\
& +\sum_{j=1}^{n} c_{i j}(t) g_{i j}\left(x_{j}\left(t-\tau_{i j}(t)\right)\right) \\
& +\sum_{j=1}^{n} d_{i j}(t) h_{i j}\left(x_{j}^{\prime}\left(t-\widehat{\tau}_{i j}(t)\right)\right) \\
& +k_{i}(t), \quad t>0, \quad i \in N .
\end{aligned}
$$

Corollary 6. Assume that $\left(H_{1}\right),\left(H_{2}\right)$, and $\left(H_{4}\right)$ hold. (33) is globally exponentially stable.

Remark 7. For autonomous system, the stability of (33) with $h_{i j}(x)=x, f_{i j}=g_{i j}$, is considered in [10]. However, the authors assume that $f_{i j}, i, j=1,2, \ldots, n$, are monotonic, bounded and $\tau_{i j}, i, j=1,2, \ldots, n$, are constants.

Remark 8 . The stability results about the zero solution of $x^{\prime}(t)=-b(t) x(t)+c(t) x(t-\tau(t))+d(t) x^{\prime}(t-\tau(t))$ are obtained by the fixed-point theory in [15]. But the differentiability of $\tau$ is needed.

\section{An Illustrative Example}

To show the effectiveness of Theorem 4, consider the following nonautonomous neural networks with impulse:

$$
\begin{aligned}
& \dot{x}_{i}(t)=-b_{i}(t) x_{i}(t)+\sum_{j=1}^{2} a_{i j}(t) f_{i j}\left(x_{j}(t)\right) \\
&+\sum_{j=1}^{2} c_{i j}(t) g_{i j}\left(x_{j}\left(t-\tau_{i j}(t)\right)\right) \\
&+\sum_{j=1}^{2} d_{i j}(t) h_{i j}\left(x_{j}^{\prime}\left(t-\widehat{\tau}_{i j}(t)\right)\right) \\
&+k_{i}(t), \quad \text { a.e. } t>0, \\
& x_{i}\left(t_{k}^{+}\right)=g_{i} x_{i}\left(t_{k}\right)+I_{i}, \\
& t_{k}=5 k, \quad i=1,2 ; \quad k=1,2, \ldots,
\end{aligned}
$$

where

$$
\begin{gathered}
\left(\begin{array}{l}
b_{1}(t) \\
b_{2}(t)
\end{array}\right)=\left(\begin{array}{l}
7+\sin t \\
5-\cos t
\end{array}\right), \quad\left(\begin{array}{l}
k_{1}(t) \\
k_{2}(t)
\end{array}\right)=\left(\begin{array}{c}
e^{-t} \\
e^{-2 t}
\end{array}\right), \\
\left(\begin{array}{l}
g_{1} \\
g_{2}
\end{array}\right)=\left(\begin{array}{l}
0.6 \\
0.3
\end{array}\right), \quad\left(\begin{array}{l}
I_{1} \\
I_{2}
\end{array}\right)=\left(\begin{array}{c}
0.3 \\
-0.1
\end{array}\right), \\
\left(a_{i j}(t)\right)_{2 \times 2}=\left(\begin{array}{cc}
0, & \frac{1}{3} \cos 3 t \\
\frac{\cos 2 t}{2}, & 0
\end{array}\right), \\
\left(c_{i j}(t)\right)_{2 \times 2}=\left(\begin{array}{cc}
\sin 2 t, & 0 \\
0, & \frac{\cos t}{2}
\end{array}\right), \\
\left(d_{i j}(t)\right)_{2 \times 2}=\left(\begin{array}{cc}
\frac{1}{6} \sin 3 t, & \frac{1}{8} \sin t \\
\frac{1}{9} \cos t, & \frac{1}{10} \cos 2 t
\end{array}\right),
\end{gathered}
$$




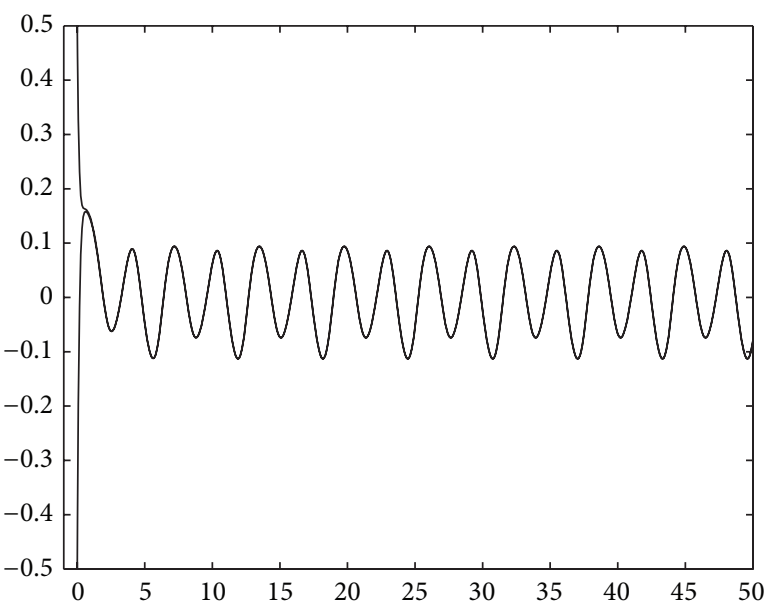

(a)

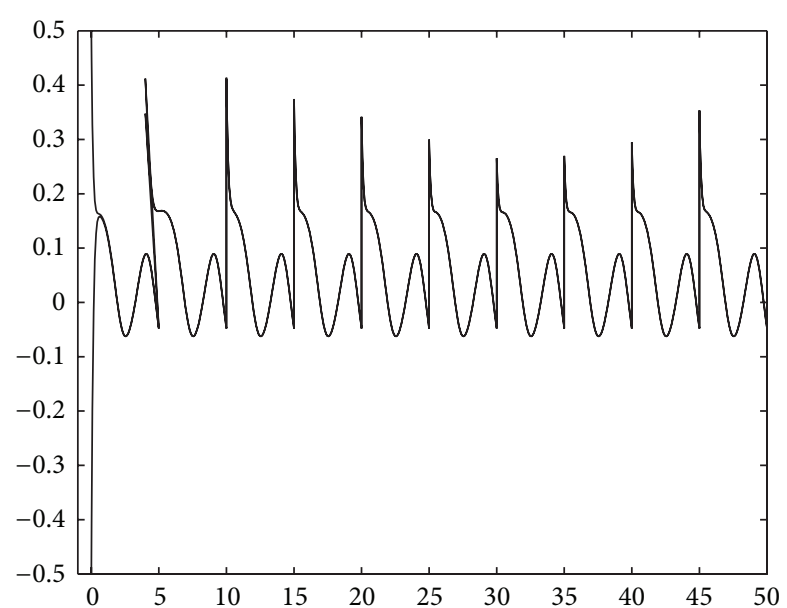

(b)

FIGURE 1: (a) Time response of state variables $x_{1}, u_{1}$ without impulsive effects. (b) Time response of state variables $x_{1}, u_{1}$ with impulsive effects.

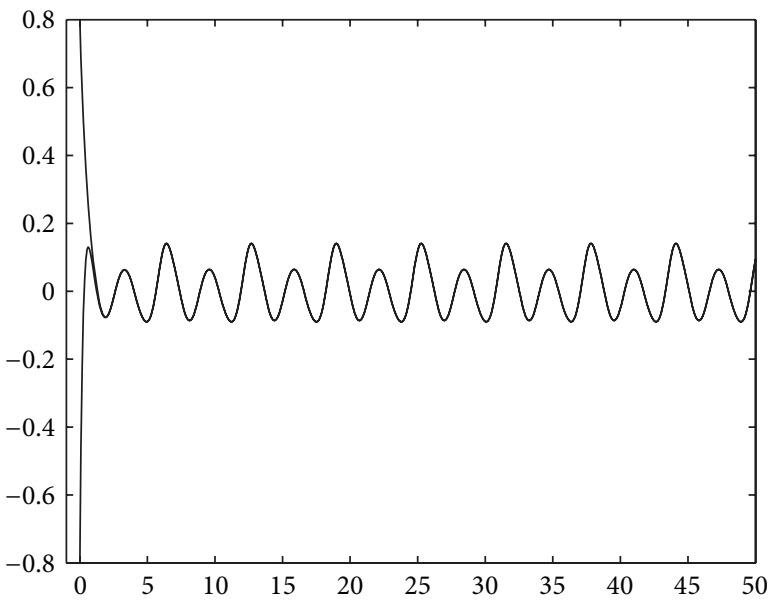

(a)

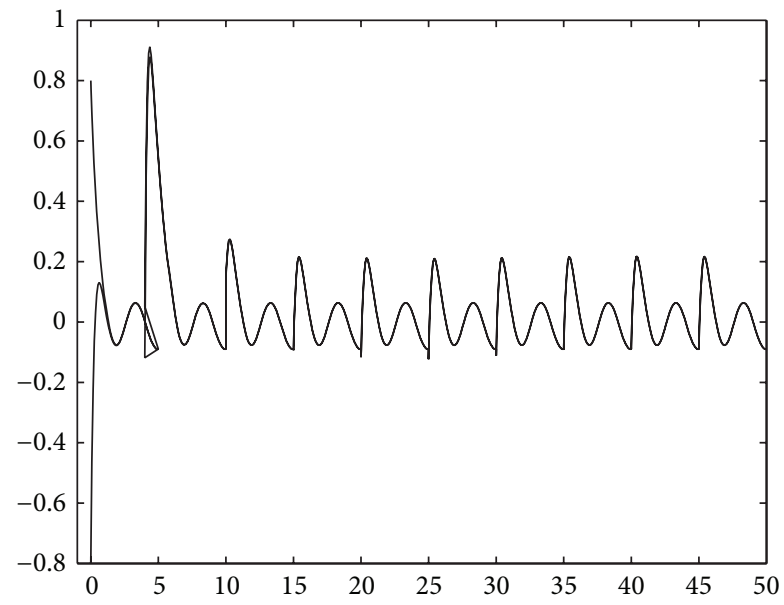

(b)

Figure 2: (a) Time response of state variables $x_{2}, u_{2}$ without impulsive effects. (b) Time response of state variables $x_{2}, u_{2}$ with impulsive effects.

$$
\begin{gathered}
\left(f_{i j}(x)\right)_{2 \times 2}=\left(\begin{array}{cc}
0, & \frac{|x+1|-|x-1|}{2} \\
\frac{|x+1|+|x-1|}{2}, & 0
\end{array}\right), \\
\left(g_{i j}(x)\right)_{2 \times 2}=\left(\begin{array}{cc}
\frac{|x+1|+|x-1|}{3}, & 0 \\
0, & \frac{|x+1|-|x-1|}{3}
\end{array}\right), \\
\left(h_{i j}(x)\right)_{2 \times 2}=\left(\begin{array}{cc}
\sin x, & \cos x \\
\cos x, & \sin x
\end{array}\right), \\
\left(\tau_{i j}(t)\right)_{2 \times 2}=\left(\begin{array}{cc}
2 \sin ^{2} t, & 0 \\
0, & 2|\cos t|
\end{array}\right),
\end{gathered}
$$$$
\left(\widehat{\tau}_{i j}(t)\right)_{2 \times 2}=\left(\begin{array}{cc}
0, & \frac{1-\sin t}{2} \\
\frac{1+\cos t}{2}, & 0
\end{array}\right) .
$$

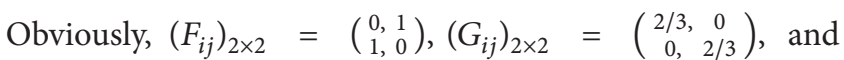
$\left(H_{i j}\right)_{2 \times 2}=\left(\begin{array}{c}1,1 \\ 1,1\end{array}\right)$.

Let $p_{1}=p_{2}=1$ and $q_{1}=18, q_{2}=10$. From the above assumption, the conditions of Theorem 4 are satisfied. Therefore, (34a) and (34b) are globally exponentially stable. $\left(x_{1}(t), x_{2}(t)\right)^{T}$ and $\left(u_{1}(t), u_{2}(t)\right)^{T}$ are the solutions of (34a) and $(34 \mathrm{~b})$ with $x_{1}(0)=0.5, x_{2}(0)=-0.8$ and $u_{1}(0)=$ $-0.5, u_{2}(0)=0.8$, respectively. Figures $1(\mathrm{a})$ and $1(\mathrm{~b})$ depict 


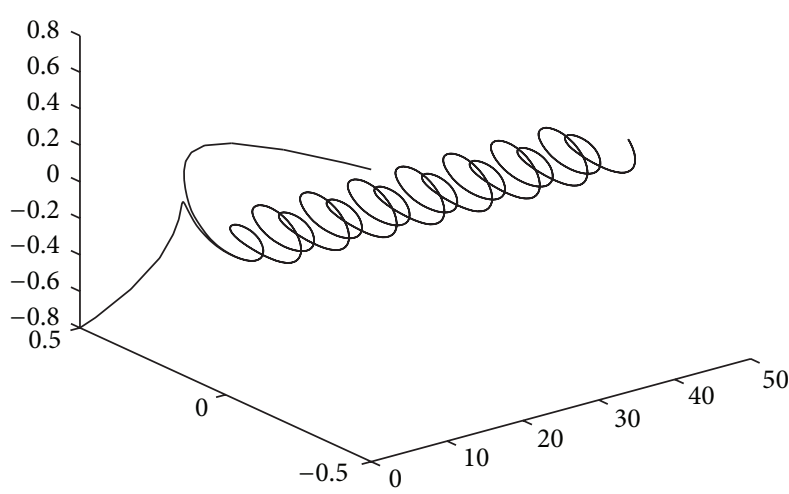

(a)

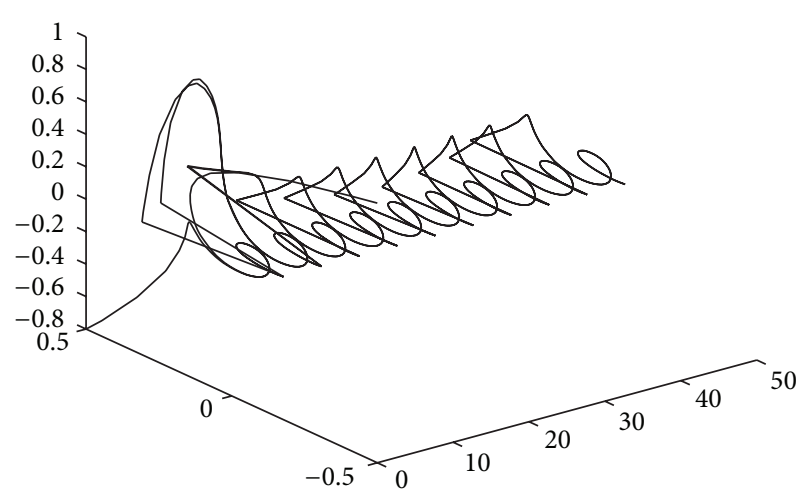

(b)

Figure 3: (a) Phase plot in space $\left(t, x_{1}, x_{2}\right),\left(t, u_{1}, u_{2}\right)$ without impulsive effects. (b) Phase plot in space $\left(t, x_{1}, x_{2}\right),\left(t, u_{1}, u_{2}\right)$ with impulsive effects.

time response of state variables $x_{1}, u_{1}$ without and with impulse effects; Figures 2(a) and 2(b) depict time response of state variables $x_{2}, u_{2}$ without and with impulse effects; Figures 3(a) and 3(b) depict the phase plot in the space $\left(t, x_{1}, x_{2}\right),\left(t, u_{1}, u_{2}\right)$ without and with impulse effects.

\section{Conflict of Interests}

The author declares that there is no conflict of interests regarding the publication of this paper.

\section{Acknowledgments}

This work was supported by the Science Foundation of Shanxi Province (no. 2010021001-1) and the National Natural Science Foundation of China (nos. 11101251 and 11001157).

\section{References}

[1] V. Lakshmikantham, D. D. Baĭnov, and P. S. Simeonov, Theory of Impulsive Differential Equations, vol. 6 of Series in Modern Applied Mathematics, World Scientific, Singapore, 1989.

[2] K. Gopalsamy, Stability and Oscillations in Delay Differential Equations of Population Dynamics, vol. 74 of Mathematics and its Applications, Kluwer Academic, Dordrecht, The Netherlands, 1992.

[3] H.-F. Huo, "Existence of positive periodic solutions of a neutral delay Lotka-Volterra system with impulses," Computers \& Mathematics with Applications, vol. 48, no. 12, pp. 1833-1846, 2004.

[4] Z. Luo and J. Shen, "Oscillation for solutions of nonlinear neutral differential equations with impulses," Computers \& Mathematics with Applications, vol. 42, no. 10-11, pp. 1285-1292, 2001.

[5] J. R. Graef, J. H. Shen, and I. P. Stavroulakis, "Oscillation of impulsive neutral delay differential equations," Journal of Mathematical Analysis and Applications, vol. 268, no. 1, pp. 310333, 2002.

[6] X. Liu and J. Shen, "Asymptotic behavior of solutions of impulsive neutral differential equations," Applied Mathematics Letters, vol. 12, no. 7, pp. 51-58, 1999.
[7] D. Xu, Z. Yang, and Z. Yang, "Exponential stability of nonlinear impulsive neutral differential equations with delays," Nonlinear Analysis: Theory, Methods \& Applications, vol. 67, no. 5, pp. 1426-1439, 2007.

[8] H. Chen, C. Zhu, and Y. Zhang, "A note on exponential stability for impulsive neutral stochastic partial functional differential equations," Applied Mathematics and Computation, vol. 227, no. 15, pp. 139-147, 2014.

[9] Y. Chen, A. Xue, R. Lu, and S. Zhou, "On robustly exponential stability of uncertain neutral systems with time-varying delays and nonlinear perturbations," Nonlinear Analysis: Theory, Methods \& Applications, vol. 68, no. 8, pp. 2464-2470, 2008.

[10] S. Xu, J. Lam, D. W. C. Ho, and Y. Zou, "Delay-dependent exponential stability for a class of neural networks with time delays," Journal of Computational and Applied Mathematics, vol. 183, no. 1, pp. 16-28, 2005.

[11] A. Bellen and N. Guglielmi, "Solving neutral delay differential equations with state-dependent delays," Journal of Computational and Applied Mathematics, vol. 229, no. 2, pp. 350-362, 2009.

[12] F. Jiang and J. Sun, "Asymptotic behavior of neutral delay differential equation of Euler form with constant impulsive jumps," Applied Mathematics and Computation, vol. 219, no. 19, pp. 9906-9913, 2013.

[13] G. Ballinger and X. Liu, "Existence and uniqueness results for impulsive delay differential equations," Dynamics of Continuous, Discrete and Impulsive Systems, vol. 5, no. 1-4, pp. 579-591, 1999.

[14] W. Zhao, "Dynamics of Cohen-Grossberg neural network with variable coefficients and time-varying delays," Nonlinear Analysis: Real World Applications, vol. 9, no. 3, pp. 1024-1037, 2008.

[15] Y. N. Raffoul, "Stability in neutral nonlinear differential equations with functional delays using fixed-point theory," Mathematical and Computer Modelling, vol. 40, no. 7-8, pp. 691-700, 2004. 


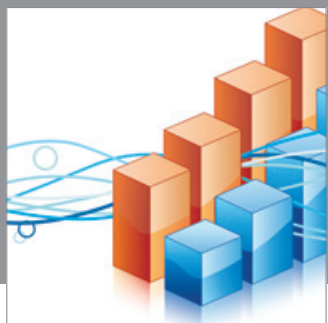

Advances in

Operations Research

mansans

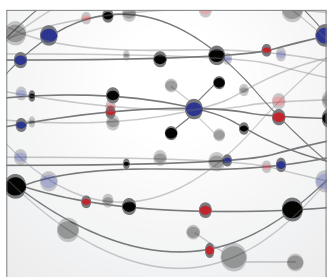

The Scientific World Journal
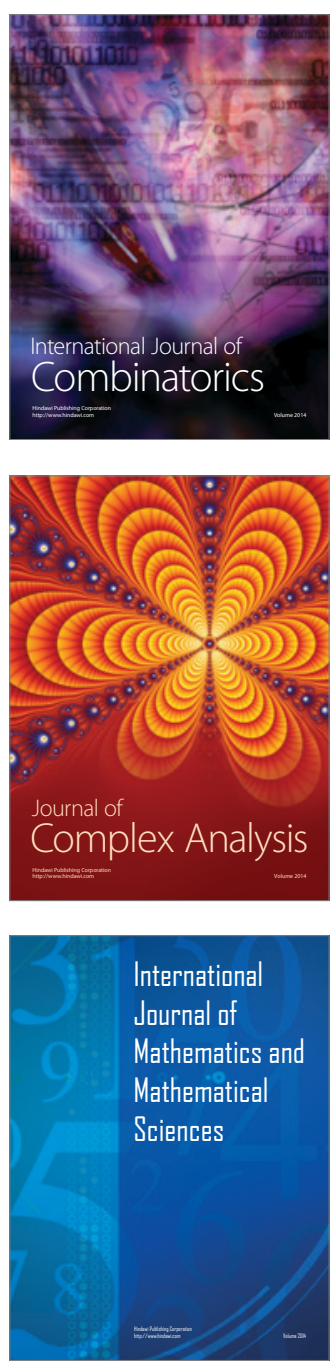
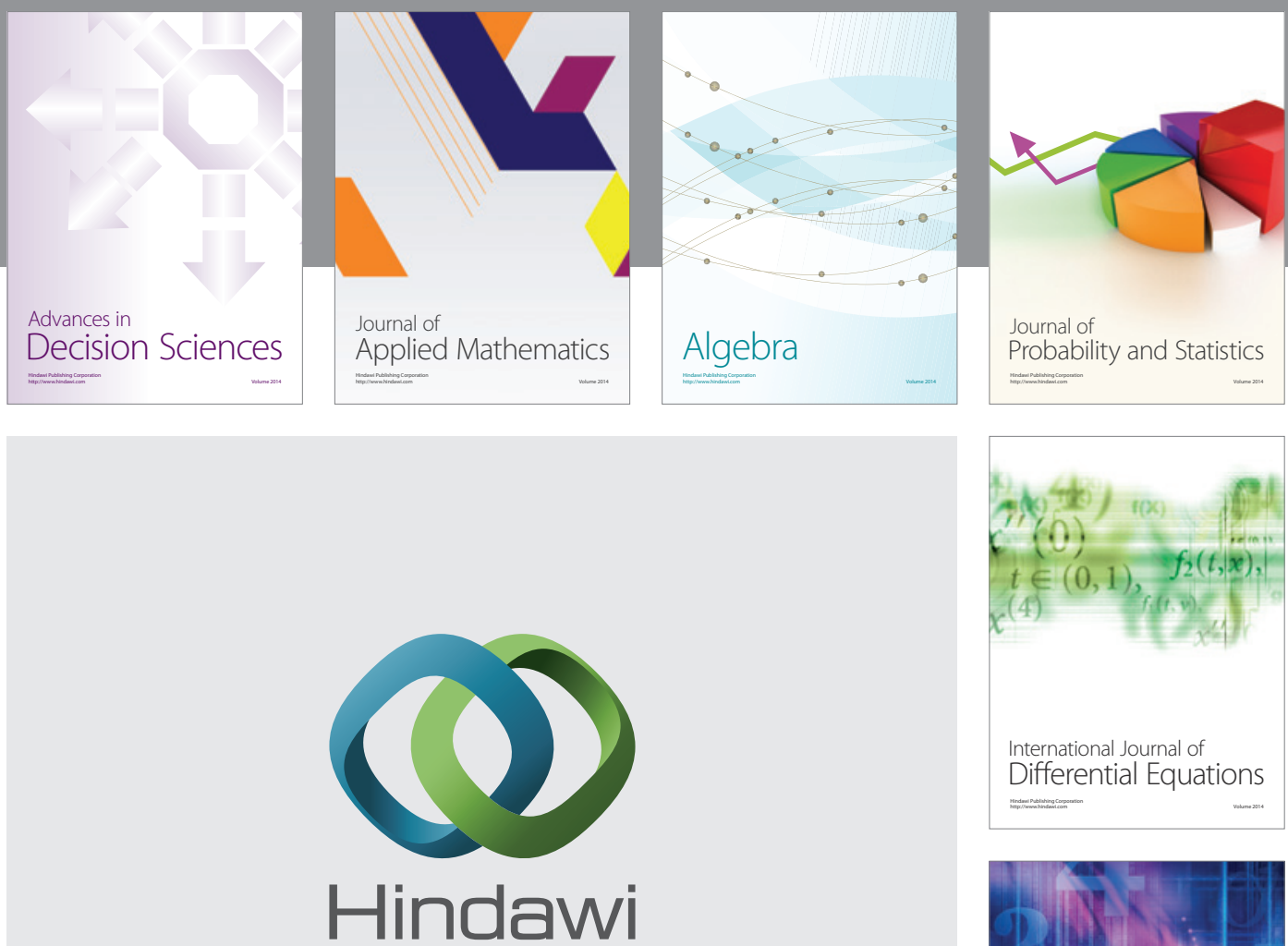

Submit your manuscripts at http://www.hindawi.com
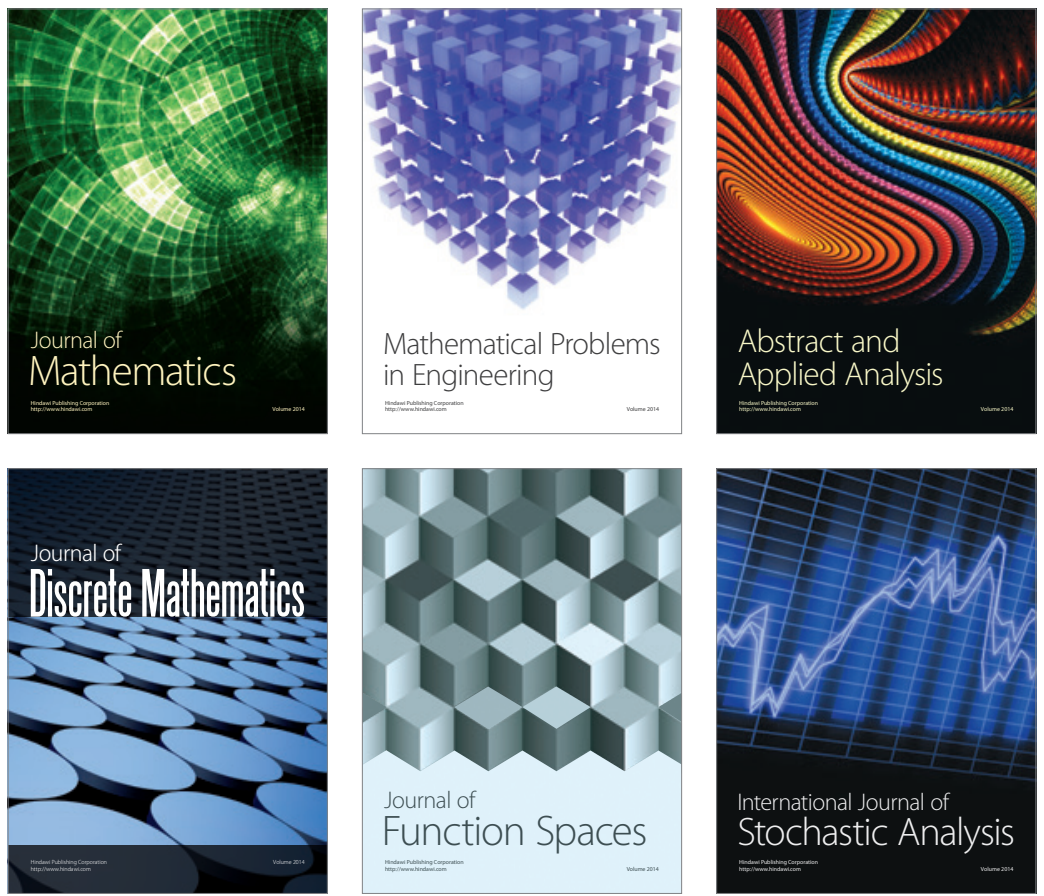

Journal of

Function Spaces

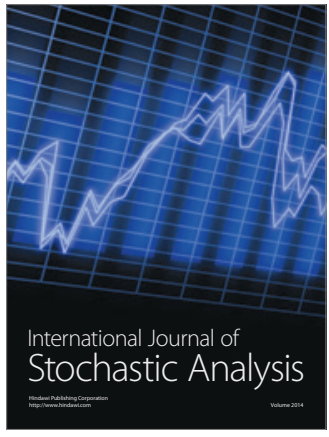

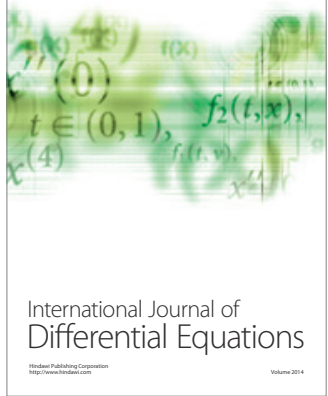
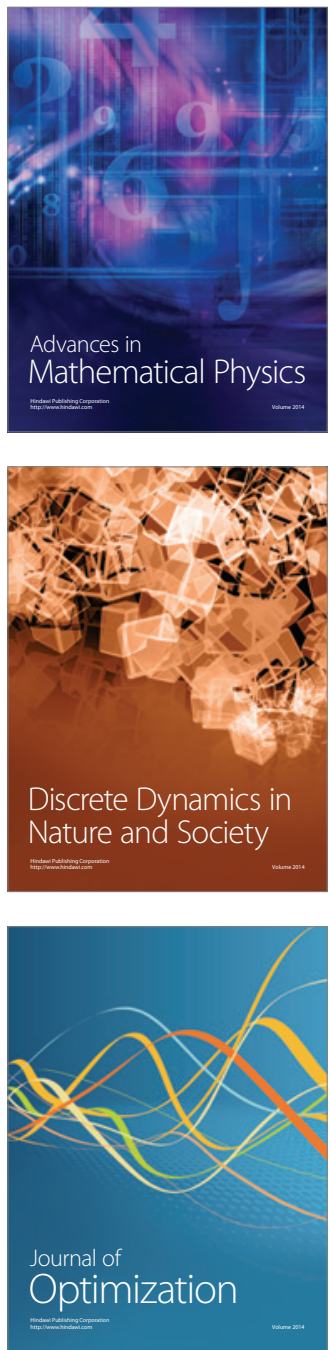